\title{
Finger Extensor Tendons
}

National Cancer Institute

\section{Source}

National Cancer Institute. Finger Extensor Tendons. NCI Thesaurus. Code C161384.

The tendons located on the dorsal side of the fingers that connect muscles of the forearm and hand to bones in the fingers, enabling straightening of the fingers. 